\title{
MY MATTER
}

Authors: Elie Ayache / Roman Vasseur

Elie Ayache

Email address: elie@ito33.com

Land address:

Bio:

Elie Ayache is a Co-Founder of ITO 33, a company providing products and services to the financial industry, and serves as its Chief Executive Officer. He has 8 years of experience as a volatility trader. He published The Blank Swan at Wiley in 2010 and The Medium of Contingency at Palgrave Macmillan in 2015.

Roman Vasseur:

Academic affiliation: Senior Lecturer, Kingston University

Land address:

Roman Vasseur,

Senior Lecturer,

School of Fine Art,

Faculty of Art, Design \& Architecture

Kingston University

Knights Park

Kingston

KT1 2QJ

Email address: r.vasseur@kingston.ac.uk 
Bio: Roman Vasseur is an artist and lecturer living and working in London, last exhibiting solo at Cubitt Gallery, in 2013. He lectures, teaches and publishes in the UK, Europe and the USA and has contributed to conferences and panel discussions at Tate Britain and the ICA. Vasseur is currently completing a practice based PhD at Goldsmith's College, London.

Abstract: An interview with the writer and financial technologist Elie Ayache by artist Roman Vasseur on the ramifications for the visual arts of Ayache's work on probability, contingency and matter in the financial markets and the implications of this work for the visual arts.

Keywords: Contingency, probability, materialism, correlationism, affect.

\section{Roman Vasseur:}

1. I've been invited to interview you as part of Martin Westwood's project Headstone to Head Drive which considers in Westwood's words: 'issues of exteriorization, technique and technology as they affect, inform and construct the 'visual' arts'. I expressed an interest in the implications that your work as a financial technologist and as a writer may have for new kinds of materialism and what implications this materiality has for arts practices and cultural production more widely. For the sake of those readers that have not read Blank Swan could you outline what the main premise of Blank Swan is with regards to probability (its end) and contingency (as a medium)?

2. You say in The Medium of Contingency (the transcript of the panel discussion held at Thomas Dane Gallery, 2011) 'the reality of the true contingent event is the same as the reality of the market. They are made of the same fabric.' [...] 'For this reason the event is real and not possible. 
It is real to the extent that it is opposed to possibility.' Perhaps you can contextualise your claims with reference to the Black, Scholes and Merton Formula for derivatives trading? This might serve to underline the role of probability in the formation of the markets over the last thirty years or so?

3. For the sake of this conversation I think what you call the 'event' has parallels with what Schmitt calls the 'decision'. A concept that Benjamin adopts in the Trauerspiel but radically alters its temporality. I think you even say that we should think of the event in terms of its impact. Can we think of the topography of the event that you describe as a groundless ground or an active form as opposed to an object form? Derrida talks in his paper The Force of Law: The Mystical Foundation of Authority of the practice of law as:

a. '[It is] the moment in which the foundation of law remains suspended in the void or over the abyss, suspended by a pure performative act that would not have to answer to or before anyone' (Derrida, 1989,991-993).

In this way the law is both situated within and anterior to its codification. This makes the doing of the law a borderline concept. Is this the ground of the 'event' you describe?

4. Could we describe this delirious deployment of probability in the markets as romantic or more specifically romanticised in the sense that it produces what cultural critic Steven Shaviro calls a modulation in which there is the appearance of freedom, liquidity and autonomy bracketed by an underlying control? I'm using Carl Schmitt's Political Romanticism here to underscore the kind of technocratic sublime that he forecast in his 1919 book as being the aestheticized, liberal ground for all political and economic life from the 1850's onwards (Schmitt, 1991). For Schmitt the political romantics main pursuit is discussion not action; discussion that 
seeks a beautiful balanced aesthetic as part of a never-ending discussion with the romantic human subject at the centre of its constellation. Is this what the markets become when they are conditioned by probability - a romanticised economy with human thought at the centre of its constellation?

5. Shaviro talks of digital media and video production, as a process akin to the probabilistic models of pricing in the markets, in that infinite variations are perceived in video but remain tethered to an underlying system of control. He employs the Grace Jones video for her track Corporate Cannibal (2008) to describe a means of identifying with the workings of capital in such a way that revivifies the reifications of finance capital and leads to a torsion. The video appears to turn Jones' body into a viscous, abstract liquid. Shaviro's assertion is that Jones is able to identify so absolutely with digital image production that she becomes an iteration that affirms video's capacity to produce a space internal to its own processes as opposed to being tied to any indexical referents including that of the human. She becomes according to Shaviro a thing, a flow of pure affect. Is this a viable aesthetic strategy for a departure from probabilistic systems that place the human and human thought at their core and as their ground?

\section{Elie Ayache:}

There is nothing romantic and nothing sublime in what I am trying to do. The title of my first book, The Blank Swan, may have relied on romantic imagery, and its subtitle, The End of Probability, on the sublime; in my new book, however, I only consider matter, the tangible and well defined matter that my prosaic industry, contingent claims and their market, is in charge of processing (Ayache,2010), (Ayache, 2015) . Of course, it is not matter similar to the one that you discover in nature or by mining the soils, and a large part of saying how tangible it is and how real it is, is pure metaphysical speculation. The peculiar matter that I analyze in The Medium of Contingency is purely metaphysically deduced and defined. Consequently, there are, in this book, considerations 
pertaining to the makeup of thought and to the meaning of things; there are general questions about time and possibility, about state and writing. In this sense, The Medium of Contingency is indeed the continuation and even the deepening of the metaphysical project of The Blank Swan. It is true that digging the structure that underlies thought in general, and the thinking of time and possibility in the particular case, might appeal to thinkers outside the strict realm of financial markets, but I insist that my attempt at digging deeper, in the present instance, has no other purpose, this time perhaps even more significantly so than the previous time, than closing my subject on itself and excluding from it anybody, really, who might shun the originality, the aridity and the particular difficulty of my topic.

I am only reporting a specialist's problem, or worse, a technical problem, whose origin is the Black-Scholes-Merton model. I am only interested in solving this problem, or in establishing the framework in which it can properly be posed, and I honestly don't know how the solution, or the reformulation of the general categories of thought that I propose on my way to the solution, can be transposed to other domains. For sure, my problem is concerned with money, and it is true that money can be a figure of the financial sublime; it is true that money can be romantic or romanticized; however, I fear that money, in my particular case, is a very specific and technical thing. Financial derivatives are settled in money - in this, they are comparable to other lotteries with financial outcomes - however, in my particular case, they are, on top of that, triggered by a tradable asset, that is to say, by an asset that is itself, at any time, fungible in money. The existence of money at both ends of my problem is what distinguishes it and, at the same time, seals it off.

I insist that my whole speculation concerning the end of probability and the end of the corresponding schema of prevision is really concerned with derivatives that are written on a tradable underlying asset and not with derivatives in general (for instance, weather derivatives, CDOs or works of art). When I argue 
that the market is the medium of contingency and is in direct contact with the event; when I declare that the market is made of the same matter, or fabric, as the future or as the event; when I say that the market takes over the event, or takes the place in which the event takes place, or that anybody who is immersed in the market, as a trader in its pit, is not predicting the event or dealing with it from a distance in time, but is situated right in the middle of the event, writing it, so to speak, exercising craft rather than knowledge, producing work rather than dwelling in a state; when I propose this revolution of metaphysics, the market to me is not just the market where stuff trades freely and continuously, but it is the bottomless pit in which the whole infinite chain of derivatives written upon derivatives all trade and admit of prices.

Crucially, the trigger of my argument is the BSM model and its dynamic replication argument, which requires the existence of prices both of the underlying asset and of the derivative written on it, and thus produces the infinite chain recursively.

I contend that there is no meaning for the price of a traded asset separately from the notion of the volatility of that price, and that there is no translation of that volatility other than by the market price of a derivative written on that asset. Volatility is a statistical concept and, in theory, you need an extended time series of prices of the underlying asset in order to estimate it; yet the advent and the practice of BSM have made it so that the volatility of the underlying asset price is inferable from the single traded price of the derivative written on it, by inversion of the BSM formula. Instead of asking a statistician or an econometrician to hand us the volatility of the underlying asset price in order to use it as an input in the BSM formula and get the value of the option as an output, we read the option price from the options market, and we invert the BSM formula against it, in order to imply (or reverse-engineer) the value the volatility must have, which we subsequently call implied volatility. 
It is not an artifact of the formalism that the volatility should be implied from the single option price instead of the extended time series of the underlying price. The reality of the option market price is an alternative reality to statistics. Instead of stepping out of the formalism in the usual direction and encountering the notion of the random generator, which we believe is lying behind the underlying prices, we step out in the opposite direction, in which the derivative price is the only 'random generator' of the underlying price, and in which the latter no longer follows a stochastic or temporal process but follows, instead, its writing as a derivative, whose price follows, in turn, its writing as a derivative of next level. A price series, indeed, of derivatives of increasing complexity, all immersed in the market, all composing a single compact matter, instead of a time series of prices of a single underlying asset. A very difficult thought, indeed; for it is difficult to realize that time is not the only perspective. This shift of perspective is truly metaphysical and it won't change the physical world or physical reality. All that one can observe, in physical time and physical reality, will still be the single register in which both the underlying asset and the derivative trade empirically and record the time series of their prices - a register in which the majority of quantitative analysts are still trapped. However, this shift of perspective will have major consequences on the notions of prediction and on our ways of dealing with the future and with the event, consequences which we will have to draw in full in order to show the extraordinary matter or fabric that the market is made of.

Precisely my contention is that the market is the matter of metaphysics and metaphysical theory, and not of physics. The matter and the reality of the market are metaphysically deduced, I said. It is not so much the empirical nature or the empirical existence of the market that really matters, but, rather, the way we think of it. The market should be approached in the same way as we approach a book, or an entity in which thought claims a constitutive part. This is a new kind of genesis, a new kind of reality altogether, which entertains an unusual link with theory and fiction and necessitates a whole new kind of philosophical analysis. I am not saying that the market is a fabrication of man, and as a consequence, 
should only be left to anthropologists or to sociologists to analyze its matter and spell out its ontology. Rather, the market deals with the event and with the future, which are real but are perspectival and engage man in their making rather than in their detached or 'objective' observation. Neither am I suggesting that the market is a matter of psychology or subjective belief. It is of no help to say that the market is an aggregate of subjective beliefs, for that vision of the market also occurs outside and uses an ill-defined notion, the belief, to compose an even more ill-defined notion, the aggregate of beliefs. The market is a trading arena, in which anything can happen and in which the trader faces, at any instant, the abyss of the event.

It has always been the concern of the philosophy of the event to say what the event really is, to face it, in a way. The event is the unprecedented, the unheard of, the unforeseen, etc., but how, then, could we speak of it, how could we recount the unaccountable and make sense of the unconceivable? How could we stare at the abyss? The event is rejected by the ontology (Badiou). We all know that the event cannot be quantified and reduced to quantitative models - that it cannot be predicted - and we all know that the event happens anywhere, anytime, in proportions, both historical and geographical, that overwhelm the market and the marketplace, so how could I be locating the event in the narrowest and most quantitative of all places, which is the trading pit? Precisely, my contention is that because the market is quantitative yet is not (in great parts, thanks to the derivatives, as we shall see) reducible to numbers and to the random generation of numbers, because it is a narrow place, even a pit, yet a place in which the whole of history and the whole stream of events pour, there will result from this extraordinary magmatic pressure an exceptional matter or compound which eventually mediates contingency and the event, a matter extending beyond physics, properly metaphysical. It is true that the trader, in the pit, seems to face, at any time, no greater an event than the up or down movement of the price and the magnitude of that movement, and it is true that, because of that seemingly elementary event, the tendency has been to look at the market exclusively through the quantitative lenses of statistics or probability. In 
reality, what this next movement of price contains is the whole event and the whole abyss, because it contains, as we shall see, the virtual exchange and the virtual pricing of all the derivatives that are virtually written on the underlying asset, and successively, one upon the other.

I said that the price, even the price of the most basic asset, has no meaning separately from its volatility. The price of an asset, trading freely in an open and frictionless exchange, is a price only insomuch as the traders exchange the asset in anticipation of its future rise or fall, and only insomuch as those future movements are in themselves totally unpredictable. For if a future price were predictable, the asset wouldn't trade at that price in the future but on the spot, by the sheer competition between traders, leaving for the future only the unpredictable. So volatility becomes the only certain thing and the only conclusion; volatility becomes the only fundamental value; volatility becomes the concept of the market and the true stuff the market is made of. Suddenly, science and quantitative theory move from trying to determine the value of the traded asset, which is impossible because the only thing that exists is its market price, to conceiving and fixing in mind the volatility of its price. Suddenly, science rules: let volatility be $\sigma$. BSM is the mathematical consequence of Brownian motion with volatility $\sigma$, and what is truly amazing is that, for the first time in the history of economics and finance, the value of something is calculated and therefore determined, through BSM. In an environment where nobody knows the value of anything - for otherwise, there wouldn't exist a market - it now suffices to recognize the existence of the market, which is tantamount to recognizing volatility, in order to determine the value of something: the value of the derivative! Note that this has nothing to do with knowledge. Volatility is equal to the meaning of the market, we said. As such, it is a semantic certainty and it is impervious to knowledge. You may call this closure sublime.

However, when the formalism of BSM is strictly followed through, in a rigorous paper like Harrison and Pliska (1981) rather than Black and Scholes (1973), one 
finds that only the underlying asset and the pit in which it is trading are ever considered (together with the money account)(Harrison \& Pliska, 1981, 215260) (Black \& Scholes, 1973, 637-654). No independently written derivative has ever been in sight, and even less so any intention of valuing it. All that BSM establishes, strictly speaking, is the dynamic trading strategy that the trader must follow in order to 'manufacture for himself' contingent payoffs. BSM instructs the trader of the exact amount of money to invest, at the inception of the trading strategy, in the exact fraction of the underlying asset, then of the exact way of varying that fraction through trading, as time goes by and the underlying asset price moves randomly, in order to end up, at the exit time or the maturity of the strategy, with the exact amount of money that the trader had in mind, at the start, as a function of the price that would then prevail for the underlying asset - in other words, a contingent payoff. No derivative or derivatives market exists at that stage, and we are only trying to manufacture a contingent payoff, or the payoff of a derivative or contingent claim that hasn't been written yet and doesn't exist yet.

The BSM formalism is no less a conceptual ascent from the trading pit of the underlying asset than the stochastic process that we described earlier. As a matter of fact, it is a more complete conceptualization of the trading activity, because it not only represents the randomness of the price but also the variation of the size of the transaction. BSM never said what the value of the volatility was, or indicated how one would get it. Volatility is a symbol in BSM - as much a symbol as when science said: Let volatility be $\sigma$. And it is the continuation of the symbolism and of the formalism to go ahead and imagine the dynamic trading strategy whose actual unfolding depends on volatility, therefore to attach a premium to a contingent payoff.

The association of the premium with the contingent payoff is as absolute a conception and a summary of the trading activity of the underlying asset as the whole stochastic process. It is as certain and as absolute for an immersed trader 
that his market exists as it is that volatility should be $\sigma$ or that the premium to manufacture the contingent payoff should be $\pi$. Both $\sigma$ and $\pi$ are conceptualizations and belong, really, to the realm beyond the end of time. Of course, in the temporal imagination of that concept, $\sigma$ has to be equal to something or other, and so does $\pi$. However, these variations are not really quantitative; they do not belong to the realm of physical time and numbers. As a matter of fact, the true variation will be qualitative; for it is at this juncture that a true event occurs. The contingent payoff suddenly gets written as a contingent claim. The trader who is immersed in a pit, who has every conceptual and semantic certainty of his volcanic trading activity, therefore every conceptual and semantic certainty of manufacturing the payoff for an initial premium $\pi$, translates this certainty as the certainty of making the market of the corresponding contingent claim and charging for it the price $\pi$. Two transfers are made, literally two translations in space, as if an imprint was irrecoverably transferred from one material to the other. First, the contingent payoff materializes as a contingent claim and gets written as one; the writing of the contingent claim is invented (for it amounts to the same to say that the contingent payoff is guaranteed to be manufactured no matter what, and to say that it is guaranteed by writing, as a contract binding a seller, that is, as a contingent claim). Second, the premium to manufacture it is translated as a price. Contrary to the common belief, option market-makers did not step into their market-to-be with a sense of uncertainty or stochasticity of volatility. They stepped in with the certainty of their tool, even with an absolute value, what we have called the concept of trading the underlying asset. In a sense, it is much better to re-immerse the conceptual ascent, from the trading pit of the underlying asset, back into the trading pit of contingent claims written on it - for this would be going back inside the pit, completing, as we shall see, its record of the event, opening it to the event in its fullest - than to come back to the pit, only to stand by its side and produce what can only turn out to be a statistical analysis of the underlying asset price. 
A trading pit is no ordinary place; it is not a random generator, subject to statistical analysis. It may look like one from outside, once you exit its volcanic time and activity and merely consider the numerical generation of prices. But what it achieves, chiefly, is to be in constant contact with the event. The event is always negatively expressed, relatively to any frame of reference that tries to capture it or represent it. For the players engaged in a game of dice, the event is not any of the outcomes; it is that a meteorite falls on their head and kills both, thus ending the game. For the random walk or for Brownian motion, the event is not that the price should go up one tick or down one tick. The event is the disruption of the stochastic process; it is that the price should jump all of a sudden, or that the volatility should jump all of a sudden. If the trading activity is to be represented in time, as a stochastic process, the event will always be, in time, that the stochastic process that was chosen for the representation was not the right one, because it did not account for that extra-event. No matter how complex or complete the stochastic process we may have selected, it will itself exactly delineate the event of its own failure. It is impossible to write in advance the stochastic process that would take into account the possibility of all events, up to and including the end of times. We cannot write the probability distribution in which any probability distribution would occur (the throw for all times). This is because a stochastic process or a probability distribution is inevitably quantitative, and quantity is inevitably contradicted by another quantity. This is because of time and of the necessity to wait, in time, until after the event, in order to produce the backward narrative, which produces the possibilities of the event (Bergson). This makes us wonder whether the record of the end of time couldn't be had and represented in another dimension than time, if only in order to prevent the ambiguity and the continual backward narrative.

This, we believe, can be achieved by the chaining and the repetition of tokens of the qualitative event that we have described above, namely, the event of writing and trading the contingent claim after the contingent payoff has been manufactured; in other words, it can be achieved by the price series of those contingent claims of increasing complexity, instead of a time series of the 
underlying asset price. When the market-maker of contingent claims steps back inside the trading pit (and not to its side) in the extraordinary move that transforms a conceptual certainty (that of the premium to manufacture the contingent payoff) into the making of a price (for the contingent claim), he produces an event that cannot be reprocessed in the previous frame by a backward narrative. The materialization of the contingent claim, as written and priced, is of the same order as a conceptual certainty; hence it cannot mix again with the time series in which the trading of the underlying is described. The invention of the writing of the contingent claim, whose immediate consequence is its trading and pricing, is not a criticism of, say, Brownian motion, to the effect that volatility is stochastic or that there exist jumps. It is generated from outside the BSM formula, from the very 'space' in which the ascent to the conceptual level is measured. As we ascend conceptually above the pit of the underlying asset to form the concept of its trading and of its volatility, we at once re-enter it materially with the newly written contingent claim. Writing the contingent claim is the equivalent, or rather, the 'exchange value' of the semantic ascent, and its subsequent trading is at once a peristaltic re-ingestion into the trading pit, which is not a readmission of time. In fact, there has never been an outside view or a conceptual ascent. The event is unconceivable, as we said, and should have never been conceived. The medium of the event is immanent and material. We are never situated in time, but always materially in the middle of the event.

The event is the very matter that the market is made of. There never was probability in the market. There has always been the event. Probability was only considered in the formalism, in Brownian motion, then in BSM; however, the right interpretation puts us back in contact with matter, with the invention of the writing and of the trading of the contingent claims. The statistical exit, what you call a 'delirious deployment of probability', all of the algorithmic trading project that issues from it, have always been the wrong exit. Now, this whole story, which I have told you about the event and about the virtual chain of prices of contingent claims, is how I think the market should be thought. I had to get the story right, because it is the story of the event, and the event is virtual. It is not 
actual. Statistics can only gather actualities. No statistics can represent the abyss of the event. Only the market can, provided the story is told right - told in a book, not in a theory of probability. The book needs the layer of the formalism as part of its matter. This is not groundless materiality. The event is truly material.

Contingency is absolute and has to be thought independently of the frame of reference of possible states and probability. As such, it is material, because matter is absolute. A new metaphysics has probably to be invented in order to recognize how material the event and the market are; and concomitantly, how different they are from probability. When we worry that the materiality of the market might be groundless, what prior notion of ground do we have in mind? What do we think the ground might be? Probability, fundamental value? The market is the record of the future, the memory of the future. This is not groundless materiality. I don't believe any reframing or recuperation or redescription of the market can be useful. One should on the contrary focus on the very specificity of the market, of money and of price - even redefine them, in the process - in order to understand new things about the event and about time and place. This has nothing to do with 'capital', with 'finance capital' or the 'workings of finance capital'. I don't know what these expressions mean. The metaphysics of the market is not so much revolutionary in its conception as it is in its matter. I don't think this new material of thought was available to the thinkers of capitalism or to its critics. I believe that my purpose, here - my mission, even - is to try to be as specific as I can be in the framing of my problem, and as dry as I can be in the description of my matter. Let any thinker or critic or artist be attracted by the intrinsic gravity of my matter, not by the displacements of its meaning.

Trading is more fundamental than probability and doesn't need probability to frame its randomness. Enough to consider the efficient market hypothesis and the conclusion will be that the path of price has to be random at any time scale which just gives Brownian motion as the simplest case. This absolute value of the 
market of a certain asset, as we called it above, is translated into the price of the contingent claim written on that asset, and then the randomness of price of the latter, which now becomes traded, is translated in turn into the price of the next, so on and so forth, in an argument which I tried my best to transplant from time into place, from the metric of probability space into the topology of the event, from prediction and prevision into writing, from light and the whole idea of vision into matter. Money and writing are the only reason why this is so, because they entail trading. They are present both at the beginning and at the end. No event disrupts the market from outside; the market itself is the continual event. This is the reason why, contrary to probability, it is commensurate with the event.

I don't think the topology of the event that I am thus describing compares with any other event. What I am trying to achieve here, what I have called my 'mission', has a unique sort of urgency attached to it. I am tackling a technological problem that is specific to the market and redefining the metaphysical view of the market, as I go. By speaking of matter, I am even attempting a return to physics, after metaphysics. Yes, I am trying to break the ground for the new hard science of the market. I truly believe that the market of contingent claims is the technology of the future; that space is curved by matter and that there exists a direct communication line between the event and the market, which does not use the relays of probability. I am deciphering the equivalence principle - as Einstein would call it - linking the matter of contingency and the geometry of price. Who needs Grace Jones to understand Einstein?

\section{Bibliography}


Ayache, E. (2010) The Blank Swan: The End of Probability. Reprint edition. Chichester, West Sussex, U.K, John Wiley \& Sons.

Ayache, E. (2015) The medium of contingency: an inverse view of the market. New York, Palgrave Macmillan.

Harrison, J.M. \& Pliska, S.R. (1981) Martingales and stochastic integrals in the theory of continuous trading. Stochastic Processes and their Applications. [Online] 11 (3), 215-260.

Jacques Derrida (1989) FORCE OF LAW:THE MYSTICAL FOUNDATION OF AUTHORITY. In: October 1989 Cardoza Law School, Cardoza Law Review. pp. 920-1047.

Schmitt, C. (1991) Political romanticism. Cambridge, Mass., MIT Press. 